\title{
SyMmetries in Hidden Bifurcation Routes to Multiscroll Chaotic Attractors Generated By Saturated Function Series
}

\author{
Faiza ZAAMOUNE ${ }^{1}$, Tidjani MENACER ${ }^{2}$, René LOZI $I^{3, *}$, Guanrong CHEN ${ }^{4}$
}

\author{
${ }^{1}$ Department of Mathematics, University Mohamed Khider, Biskra, Algeria \\ ${ }^{2}$ Department of Mathematics, University Mohamed Khider, Biskra, Algeria \\ ${ }^{3}$ Université Côte d'Azur, CNRS, LJAD, Parc Valrose 06108, Nice Cedex 02, France \\ ${ }^{4}$ Department of Electrical Engineering, City University of Hong Kong, Hong Kong SAR, China
}

*Corresponding Author: René LOZI (email: Rene.lozi@univ-cotedazur.fr)

(Received: 01-Sep-2019; accepted: 18-Nov-2019; published: 31-Dec-2019)

DOI: http://dx.doi.org/10.25073/jaec.201934.256

\begin{abstract}
In this paper, hidden bifurcation routes to multiscroll chaotic attractors generated by saturated function series are explored. The method to find such hidden bifurcation routes (HBR) depending upon two parameters is similar to the method introduced by Menacer, et al. (2016) for Chua multiscroll attractors. These $H B R$ are characterized by the maximal range extension (MARE) of their attractors and coding the appearance order of the scrolls under the control of the two parameters. Moreover, these $H D R$ have interesting symmetries with respect to the two parameters. The novelty that this article introduces, is firstly the paradigm of MARE and the formula giving their approximate value depending upon parameters $p$ and $q$, which is linked to the size of the scrolls; secondly the coding of the HBR which is defined for the first time including the basic cell ; and thirdly unearthing the symmetries of these routes, allowing to obtain their coding without any numerical computation.
\end{abstract}

\section{Keywords}

Hidden bifurcation, Multiscroll chaotic attractor, Saturated function series, Symmetry.

\section{Introduction}

In the last three decades, generation of multiscroll chaotic attractors has been extensively studied due to their promising applications in various real-word technologies. Several methods have been proposed, like piecewise linear functions and nonlinear modulating functions, and in electronic circuits (step, hysteresis and saturated circuits), for generating multidirectional multiscroll chaotic attractors (see [1] for a survey). Although the majority of such multiscroll generations are known for many years, it is only recently that they are studied under the scope of bifurcation theory [2]. They also have been found for hidden attractors [3] in the case where equilibrium points exist [4], and even in the case of infinite number of equilibriums [5]. For all the multiscrolls already known, the number of scrolls (or spirals) is a fixed integer, which depends on 
one or more discrete parameters. To date no bifurcation was analyzed. However, Menacer et al. [2] changed the paradigm of discrete parameters by introducing hidden bifurcations, generating multiscrolls in a family of systems (7) possessing a continuous bifurcation parameter. Then, all the classical theories of dynamical systems and their powerful tools can be used for studying the multiscrolls. Such hidden bifurcation theory is based on hidden attractor theory introduced by Leonov et al. [6,7].

In this article, the focus is on the study of the symmetries of hidden bifurcation routes in 1-D multiscroll chaotic attractors generated by saturated function series. In [8], a saturated function series was proposed for generating multiscroll chaotic attractors, including 1-D $n$-scroll, 2-D $n \times m$-grid scroll, and 3-D $n \times m \times l$-grid scroll chaotic attractors. The present article will adopt saturated function series in the design.

The novelty that this article introduces, is firstly the paradigm of the maximal attractor range extension and the formula giving their approximate value depending upon parameters $p$ and $q$, which is linked to the size of the scrolls; secondly the coding of the HBR which is defined for the first time including the basic cell ; and thirdly unearthing the symmetries of these routes, allowing to obtain their coding without any numerical computation.

This article is organized as follows: In Section 2 , the model of multiscroll chaotic attractors generated by saturated function series proposed in [1] is reviewed. In Section 3, the localization method introduced in [2] is presented for hidden bifurcation in multiscroll chaotic attractors generated by saturated function series. In Section 4, this method is assessed on two numerical examples. In Section 5, the symmetries are studied, which occur in hidden bifurcation routes when two parameters vary. Finally, in Sec. 6, a brief conclusion is drawn. Appendix A presents the analytical-numerical method for hidden attractor localization proposed by Leonov [7].

\section{Multiscrool chaotic} attractors from saturated function series

Among the several proposed methods for creating $n$-scroll $(n \geq 3)$ chaotic attractors [9-11], for the one in [8], which is based on saturated function series (Fig. 1) a controller is added to a linear system

$$
\left\{\begin{array}{l}
\dot{x}=y \\
\dot{y}=z \\
\dot{z}=-a x-b y-c z+d_{1} f(x ; k ; h ; p ; q)
\end{array}\right.
$$

where

$$
f(x ; k ; h ; p ; q)=\left\{\begin{array}{lc}
y_{1, k} & \text { if } x>q h+1 \\
y_{2, k, i} & \text { if }|x-i h| \leq 1 \\
& -p \leq i \leq q \\
y_{3, k, i} & \text { if } l_{1, i}<x<l_{2, i} \\
& -p<i<q-1 \\
y_{4, k} & \text { if } x<-p h-1
\end{array}\right.
$$

with $l_{1, i}=i h+1$ and $l_{2, i}=(i+1) \times h-1$, $y_{1, k}=(2 q+1) k, y_{2, k, i}=k(x-i h)+2 i k$, $y_{3, k, i}=(2 i+1) k$ and $y_{4, k}=-(2 p+1) k$

Parameters $p, q, h$ and $k$ are integers, and $a, b, c, d_{1}$ are real numbers.

Throughout this article, set the parameter values as $a=b=c=d_{1}=0.7$. The number $n$ of scrolls (also denominated spirals elsewhere [2]) satisfies

$$
n=p+q+2
$$

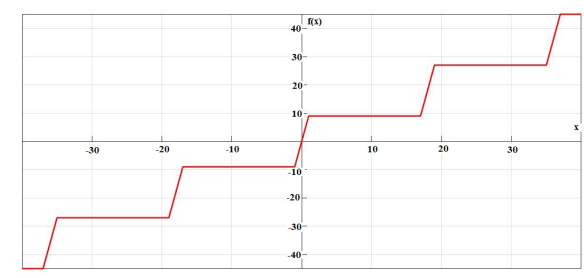

Fig. 1: Saturated function series, when $p=q=2$ and $k=9, h=18$. 


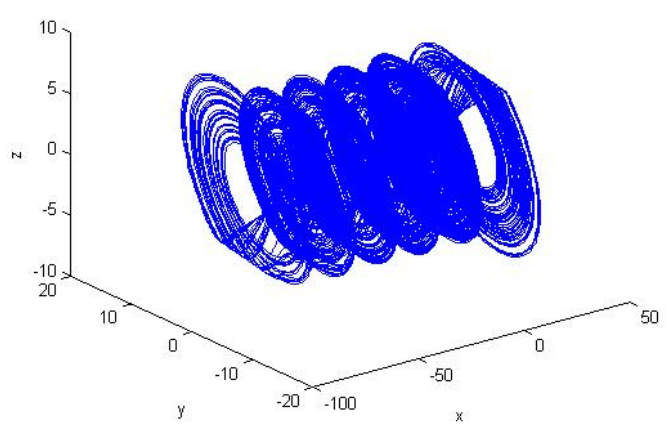

Fig. 2: The 6-spiral attractor generated by Eqs. (1) and (2), with $k=9 ; h=18, p=q=2$ and $a=b=$ $c=d_{1}=0,7$.

For $k=9, h=18, p=q=2$, a 6 -scroll attractor is generated as the asymptotic attractor of system (1-2), see Fig. 2.

\section{Recovering hidden bifurcation in a multiscrool chaotic} attractor

In the modified Chua circuit with a sine function, the parameter $c$ governing the number of spirals is an integer, hence it is not possible to vary it continuously and therefore it is not possible to observe bifurcations of attractors from $n$ to $n+2$ spirals as the parameter $c$ changes. Moreover it is not possible to use non-integer real values for $c$. To overcome this obstacle, Menacer et al. [2] introduced a new method for uncovering hidden bifurcations based on the idea of Leonov and Kuznetsov [6] for searching hidden attractors (i.e. homotopy and numerical continuation, see Appendix). While keeping $c$ constant, a new bifurcation parameter $\varepsilon$ is introduced. This method is now applied to multiscroll chaotic attractors from saturated function series. Rewrite system (1-2) to the form

$$
\frac{d X}{d t}=M X+\mu \psi\left(\nu^{T} X\right), \quad X \in \mathbb{R}^{3}
$$

with

$$
\begin{aligned}
& M=\left(\begin{array}{ccc}
0 & 1 & 0 \\
0 & 0 & 1 \\
-a & -b & -c
\end{array}\right), \quad X=\left(\begin{array}{l}
x \\
y \\
z
\end{array}\right), \\
& \nu=\left(\begin{array}{l}
1 \\
0 \\
0
\end{array}\right), \quad \mu=\left(\begin{array}{c}
0 \\
0 \\
d_{1}
\end{array}\right) \text { and } \psi(\sigma)=f(\sigma) .
\end{aligned}
$$

Introduce a coefficient $k_{0}$ and a small parameter $\varepsilon$ to represent system (4) as

$$
\frac{d X}{d t}=M_{0} X+\mu \varepsilon \varphi\left(\nu^{T} X\right),
$$

where

$$
\begin{gathered}
M_{0}=M+k_{0} \mu \nu^{T}=\left(\begin{array}{ccc}
0 & 1 & 0 \\
0 & 0 & 1 \\
k_{0} d_{1}-a & -b & -c
\end{array}\right), \\
\lambda_{1,2}^{M_{0}}= \pm i \omega_{0}, \quad \lambda_{3}^{M_{0}}=-d
\end{gathered}
$$

In practice, to determine $k_{0}$ and $\omega_{0}$, one uses the following transfer function of system (5):

$$
W_{M_{0}}(\lambda)=\nu(M-\lambda I)^{-1} q
$$

where $\lambda$ is a complex variable. The number $\omega_{0}$ is determined from the equation $\operatorname{Im} W\left(i \omega_{0}\right)=$ 0 and $k_{0}$ is calculated by the formula $k_{0}=$ $\left(\operatorname{Re} W\left(i \omega_{0}\right)\right)^{-1}$.

Using the nonsingular linear transformation $X=S Y$, defined in the Appendix, system (5) is reduced to the form

$$
\frac{d Y}{d t}=A Y+b \varepsilon \varphi\left(c^{T} Y\right)
$$

where

$$
\begin{aligned}
A & =\left(\begin{array}{ccc}
0 & -\omega_{0} & 0 \\
\omega_{0} & 0 & 0 \\
0 & 0 & -d
\end{array}\right), \quad Y=\left(\begin{array}{l}
y_{1} \\
y_{2} \\
Y_{3}
\end{array}\right), \\
b & =\left(\begin{array}{c}
b_{1} \\
b_{2} \\
1
\end{array}\right), \quad \text { and } \quad c=\left(\begin{array}{c}
1 \\
0 \\
-h
\end{array}\right) .
\end{aligned}
$$

Remark 1. System (7) is 3-dimensional, with $Y_{3} \in \mathbb{R}$. 
The transfer function of system (7) can be represented as

$$
\begin{aligned}
W_{A}(\lambda) & =C^{T}(A-I \lambda)^{-1} B \\
& =\frac{h}{d+\lambda}-\lambda \frac{b_{1}}{\lambda^{2}+\omega_{0}^{2}}+\omega_{0} \frac{b_{2}}{\lambda^{2}+\omega_{0}^{2}}
\end{aligned}
$$

Further, using the equality of transfer functions of systems (5) and (7), one obtains

$$
W_{A}(\lambda)=\nu^{T}\left(M_{0}-\lambda I\right)^{-1} \mu
$$

This implies the following relations:

$$
\begin{array}{ll}
k_{0}=\frac{a-\omega_{0}^{2} d}{d_{1}}, & d=c, \quad h=\frac{-d_{1}}{\omega_{0}^{2}+d^{2}}, \\
b_{1}=\frac{-d_{1}}{\omega_{0}^{2}+d^{2}}, & b_{2}=\frac{-c d_{1}}{\omega_{0}\left(\omega_{0}^{2}+d^{2}\right)}
\end{array}
$$

Since system (5) can be reduced to the form (7) by the non-singular linear transformation defined in Appendix, the following relationships can be obtained:

$$
A=S^{-1} M_{0} S, b=S^{-1} \mu, c^{T}=\nu^{t} S
$$

Solving these matrix equations gives the entries of this matrix:

$$
S=\left(\begin{array}{ccc}
S_{11} & S_{12} & S_{13} \\
S_{21} & S_{22} & S_{23} \\
S_{31} & S_{32} & S_{33}
\end{array}\right)
$$

with

$$
\begin{aligned}
& S_{11}=1, \quad S_{12}=0, \quad S_{13}=-h \\
& S_{21}=0, \quad S_{22}=-\omega_{0}, \quad S_{23}=d h \\
& S_{31}=-\omega_{0}^{3}, \quad S_{32}=0, \quad S_{33}=d^{2} h
\end{aligned}
$$

For small enough $\varepsilon$, one can determine the initial data of the first step in the multistage localization procedure, as

$$
X(0)=S V(0)=S\left(\begin{array}{c}
\eta_{0} \\
0 \\
0
\end{array}\right)=\left(\begin{array}{c}
\eta_{0} S_{11} \\
\eta_{0} S_{21} \\
\eta_{0} S_{31}
\end{array}\right)
$$

For system (4), this gives the initial condition $X^{0}(0)=\left(x^{0}(0)=\eta_{0}, y^{0}(0)=0, z^{0}(0)=-\eta_{0} \omega_{0}^{3}\right)$

\section{Examples and}

properties of

\section{bifurcation routes}

\subsection{Numerical computation of two hidden bifurcation routes}

The goal of this article is to study the hidden bifurcation routes of 1-D multiscroll attractors defined in Section 2 and their symmetries. First, two examples of such hidden bifurcation routes are shown, and the way in which the scrolls appear are revealed.

Consider the system (1-2) with parameter values

$$
a=b=c=d_{1}=0.7
$$

Now, the localization procedure described above is applied to system (4) with multiple spiral attractors. For this purpose, the following starting frequency $\omega_{0}$ and a coefficient of harmonic linearization $k_{0}$ are computed, as explained in the Appendix:

$$
\omega_{0}=0.8366 \text { and } k_{0}=0.3
$$

Then, the solutions of system (5), with the nonlinearity $\varepsilon \varphi(x)=\varepsilon\left(\psi(x)-k_{0} x\right)$, are computed by increasing sequentially $\varepsilon$ from the value $\varepsilon=0.1$ to $\varepsilon=1$, with step 0.1 . For $p=0, q=4$, $h=20$ and $k=10$, using (13), one obtains the initial conditions

$$
x_{0}(0)=203.2, \quad y_{0}(0)=0, \quad z_{0}(0)=-119.01
$$

whereas in the case of $p=2$ and $q=3$, with the same values of $h$ and $k$ the following initial conditions are obtained:

$$
x_{0}(0)=249, \quad y_{0}(0)=0, \quad z_{0}(0)=-145.83
$$

Using these initial conditions, the procedure described in section 3 yields the values of the parameter $\varepsilon$ at the bifurcation points, where the attractor increases the number of spirals from 1 
to 6 spirals ( 7 spirals, respectively), as shown in both Table 1, and Table 2. Note that there is a special feature or case 7 compared to the case 6 , where the bifurcations appear respectively from 1 to 7 spirals, in the following order: 1, 2, 4, 6 and 7 , depending on the values of $\varepsilon$, as shown in Figures 3, 4, 5 and 6.

\begin{tabular}{|c|c|c|}
\hline Values of $\varepsilon$ & 0.41 & 0.6 \\
\hline Number of spirals & 1 spiral & 2 spirals \\
\hline Values of $\varepsilon$ & 0.95 & 0.985 \\
\hline Number of spirals & 3 spirals & 4 spirals \\
\hline Values of $\varepsilon$ & 0.988 & 0.99 \\
\hline Number of spirals & 5 spirals & 6 spirals \\
\hline
\end{tabular}

Tab. 1: Values of the parameter $\varepsilon$ at the bifurcation points for $p=0$ and $q=4$ ( 6 scrolls $)$.

\begin{tabular}{|c|c|c|}
\hline Values of $\varepsilon$ & 0.42 & 0.6 \\
\hline Number of spirals & 1 spiral & 2 spirals \\
\hline Values of $\varepsilon$ & 0.95 & 0.98 \\
\hline Number of spirals & 4 spirals & 6 spirals \\
\hline Values of $\varepsilon$ & 0.99 & \\
\hline Number of spirals & 7 spirals & \\
\hline
\end{tabular}

Tab. 2: Values of the parameter $\varepsilon$ at the bifurcation points for $p=2$ and $q=3$ ( 7 scrolls).

\subsection{Maximal attractor range extension and coding order of scrolls appearance}

Both Tables 1 and 2 summarize the appearance of scrolls versus the values of $\varepsilon$. Figs. 3 to 5 display some interesting information: the order of scroll appearance and the maximal attractor range extension. In both routes, the parameter values of function (2) are $k=10$ and $h=20$. These parameters play a significant role in the sizes of the attractors.

The maximal attractor range extension $\left(M A R E_{p, q}\right)$ is the size of the $x$-projection of the considered attractor defined by parameter values $p$ and $q$, when $\varepsilon=1$ and as $t \rightarrow+\infty$. For example, for the first route defined in Table 1, one can see from Fig. 4 that the minimum

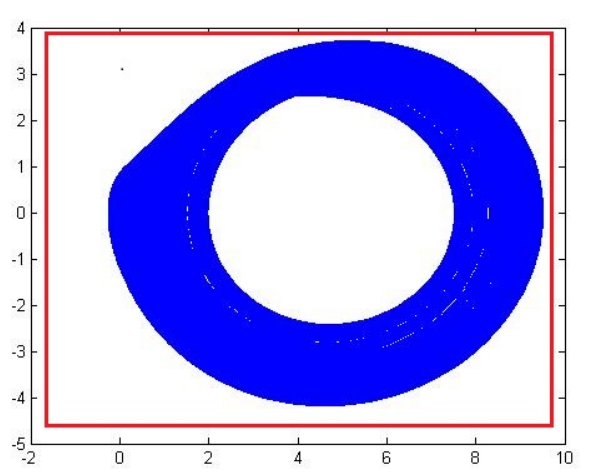

(a)

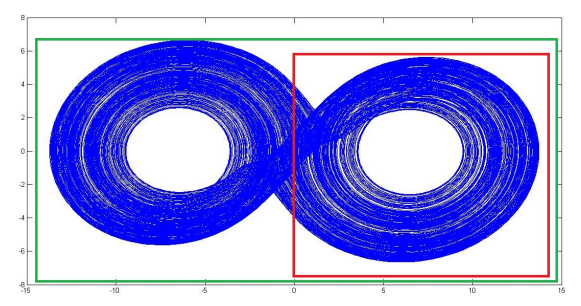

(b)

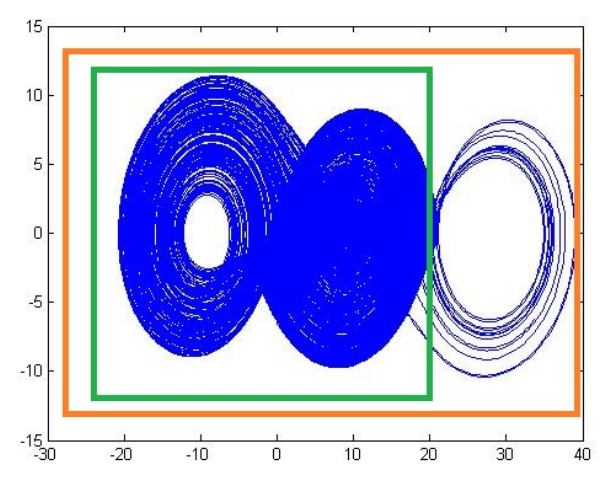

(c)

Fig. 3: The increasing number of spirals of system (5) according to increasing $\varepsilon$ values, when $p=0$ and $q=4, k=10$ and $h=20$. (a) : The first scroll between 0 and 20 for $\varepsilon=0.41$, (b) : The second scroll on the left for $\varepsilon=0.6$, (c) : The third scroll on the right for $\varepsilon=0.95$. The horizontal axis is the $x$-axis, the vertical axis is the $y$-axis.

value of the range of the variable $x$ of the attractor is -20 , and the maximum value is 100 . 


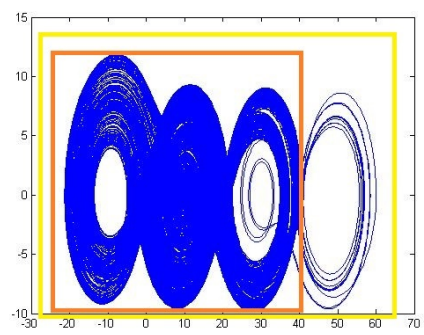

(a)

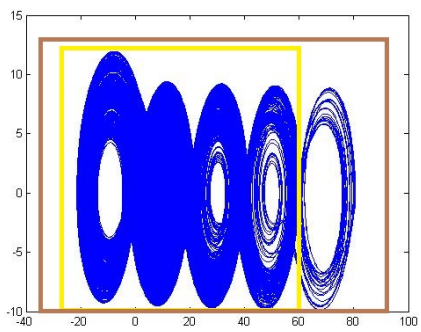

(b)

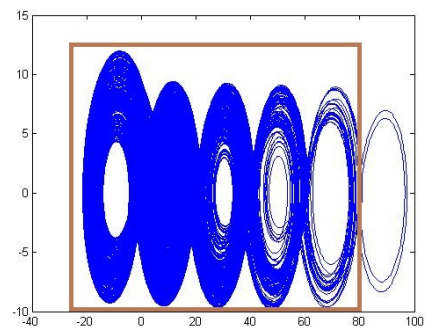

(c)

Fig. 4: The increasing number of spirals of system (5) according to increasing $\varepsilon$ values, when $p=0$ and $q=4$, $k=10$ and $h=20$. (a) : The fourth scroll on the right for $\varepsilon=0.985$, (b) : The fifth scroll on the right for $\varepsilon=0.988$, (c) : The sixth scroll on the right for $\varepsilon=0.99$. The horizontal axis is the $x$-axis, the vertical axis is the $y$-axis.

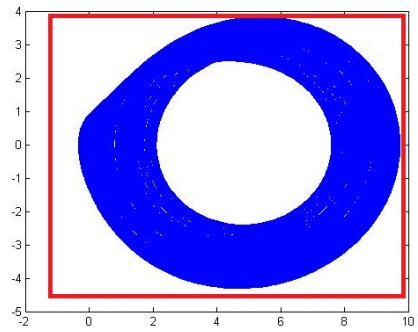

(a)

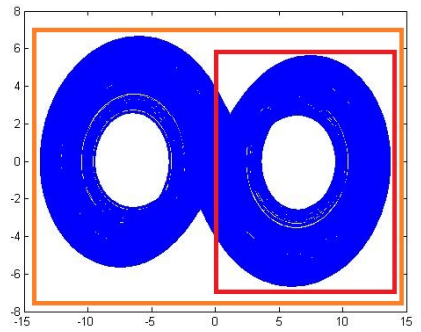

(b)

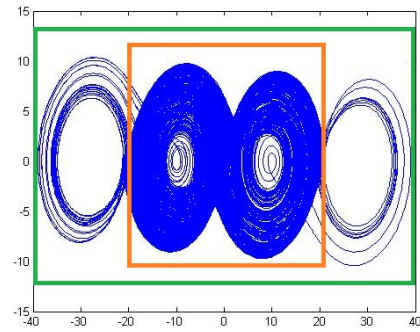

(c)

Fig. 5: The increasing number of spirals of system (5) according to increasing $\varepsilon$ values, when $p=2$ and $q=3$, $k=10$ and $h=20$. (a) : The first scroll between 0 and 20 for $\varepsilon=0.42$, (b) : The second scroll on the left for $\varepsilon=0.6$, (c) : The third and the fourth scrolls: two left-right symmetrical for $\varepsilon=0.95$. The horizontal axis is the $x$-axis, the vertical axis is the $y$-axis.

Therefore, in this case, $M A R E_{0,4}=[-20,100]$, and its length is equal to 120 for 6 scrolls. For the second route in Table 2, the attractor spans between -60 and 80 (Fig. 6) having $M A R E_{2,3}=[-60,80]$ with a length equa1 to 140 for 7 scrolls. In both cases, the length of MARE is equal to the number of scrolls $\times 20$ (i.e. $(n+p+2) \times 20$, following $(3))$.

Moreover, when $\varepsilon$ increases (Figs. 3 and 4 ), the size of each scroll is expanding. It is approximatively equal to $(17 \times \varepsilon)+3$. By defining the interval $[v \times 20, w \times 20]^{\{\varepsilon\}}$ as

$[v \times 20, w \times 20]^{\{\varepsilon\}}=[v \times(17 \times \varepsilon+3), w \times(17 \times \varepsilon+3)]$ the first scroll fulfills the interval $[0,20]^{\{\varepsilon\}}$. The second scroll appears symmetrically to the first one and fulfills the interval $[-20,20]^{\{\varepsilon\}}$. Now, introduce the coding $[0,20]^{\{\varepsilon\}} / L$ (L stands for the left of the previous interval) to designate the progression of such appearance of scrolls. After the appearance of the second scroll, the third one belongs to the interval $[20,40]^{\{\varepsilon\}}$, the fourth to the interval $[40,60]^{\{\varepsilon\}}$, the fifth to the interval $[60,80]^{\{\varepsilon\}}$, and the last to the interval $[80,100]^{\{\varepsilon\}}$.

The coding of this hidden bifurcation route $(H B R)$, which ends with the interval $[-20,100]^{\{\varepsilon\}}$, is $H B R_{0,4}=$ $[0,20]^{\{\varepsilon\}} / L / R / R / R / R(R$ stands for the right 


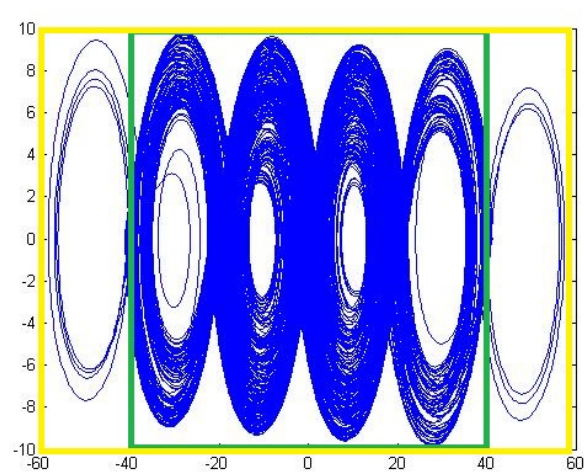

(a)

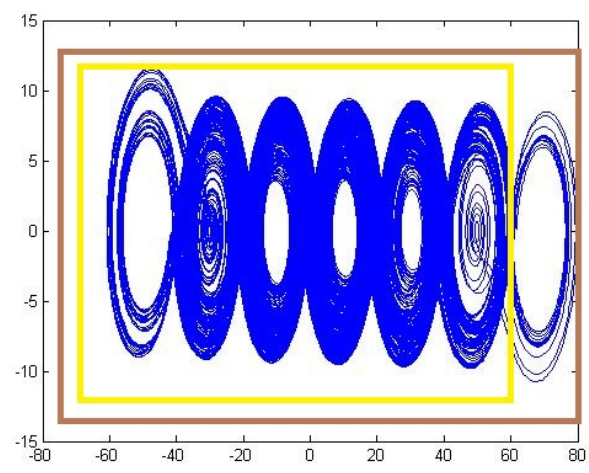

(b)

Fig. 6: The increasing number of spirals of system (5) according to increasing $\varepsilon$ values, when $p=2$ and $q=3, k=10$ and $h=20$. (a) : The fifth and the sixth scrolls: two symmetrical left-right for $\varepsilon=0.98$, (b) : The seventh scroll on the right for $\varepsilon=0.99$. The horizontal axis is the $x$-axis, the vertical axis is the $y$-axis.

of the previous interval).

As the value of $\varepsilon$ is not important for the search of symmetries of the hidden bifurcation routes, it is omitted and denote simply $H B R_{0,4}=[0,20]^{\{\varepsilon\}} / L / R / R / R / R$ by $H B R_{0,4}=[0,20] / L / R / R / R / R$.

The beginning of the second route (Figs. 5 and $6)$ is the same $\left([0,20]^{\{\varepsilon\}} / L\right)$. However, the third and the fourth scrolls appear simultaneously, expanding the interval $[-20,20]^{\{\varepsilon\}}$ to $[-40,40]^{\varepsilon}$. Denote this expansion by $[0,20]^{\{\varepsilon\}} / L / 2$ Sym . The fifth and the sixth scrolls appear again symmetrically to the previous attractor. Finally, the last scroll belongs to the interval $[60,80]^{\{\varepsilon\}}$.

The coding of this second route, which ends with the interval $[-60,80]^{\{\varepsilon\}}$, is $H_{B R} R_{2,3}=[0,20]^{\{\varepsilon\}} / L / 2 S y m / 2 S y m / R$ or simply $H_{B R} R_{2,3}=[0,20] / L / 2 S^{2} m^{2} / R$.

\section{Symmetries of the hidden bifurcation \\ routes}

Consider all the values of $p$ and $q$ for the values of $n$ in Eq. (3), ranging from 3 to 7, and some values of $p$ and $q$ for $n$ between 8 and 12 .

\subsection{Basic cell}

The numerical experiments show that the first two scrolls appear following either the coding

$$
\left([0,20]^{\{\varepsilon\}} / L\right) \text { or }\left([-20,0]^{\{\varepsilon\}} / R\right)
$$

as displayed in Figs. 7 and 8, for the same values of $h$ and $k$. Therefore, it is called the basic cell and denoted as $B$, either

$$
\left([0,20]^{\{\varepsilon\}} / L\right) \text { or }\left([-20,0]^{\{\varepsilon\}} / R\right)
$$

Together with $B$, the following generalized notations are used for the coding:

$$
\begin{gathered}
2 \text { Sym }^{s}=\underbrace{2 \text { Sym } / 2 S y m / \ldots / 2 S y m}_{\text {stimes }} \\
L^{t}=\underbrace{L / L / \ldots / L}_{t \text { times }} \\
R^{u}=\underbrace{R / R / \ldots / R}_{u \text { times }}
\end{gathered}
$$

\subsection{Symmetries}

All the hidden bifurcation routes have been computed numerically for all the values of $p$ and $q$, giving the values of $n$ in Eq. (3) ranging from 3 to 7 . The results (MARE and coded bifurcation 


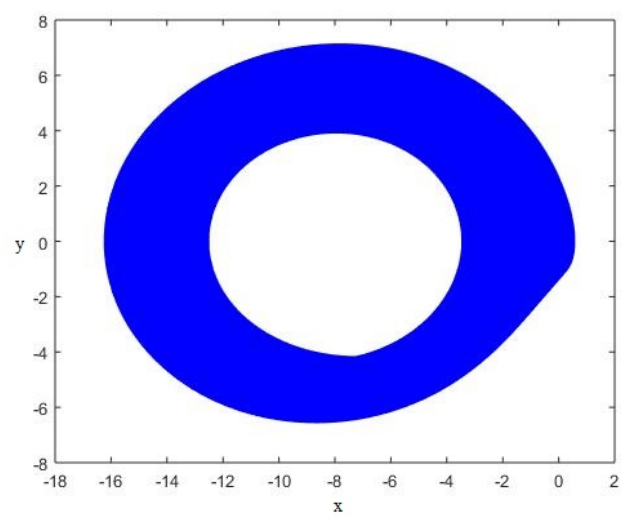

Fig. 7: The first scroll between -16 and 0 for the values of the parameters $p=0$ and $q=4$ with the parameters values $k=9$ and $h=18$.

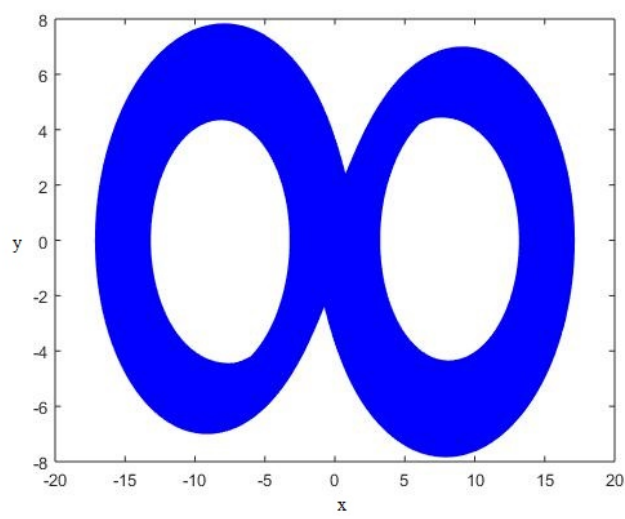

Fig. 8: The second scroll is in symmetry with the first one, generated between 0 and $20(-20,0)$ for the values of the parameters $p=0$ and $q=4$ with the parameters values $k=9$ and $h=18$.

routes) are displayed in black color in Table 3. It was found that

$$
M A R E_{p, q}=[-20-20 \times p, 20+20 \times q]
$$

Moreover the coding of every hidden bifurcation route is given by

$$
H B R_{p, q}== \begin{cases}B / 2 \text { Sym }^{s} & \text { if } p=q \\ B / 2 \text { Sym }^{s} / L^{p-s} & \text { if } p>q \\ B / 2 \text { Sym }^{s} / R^{q-s} & \text { if } p<q\end{cases}
$$

which is the formula that determines the number of scrolls and the order of their appearances, where $s=\min (p, q)$.
In Table $3, H B R_{p, q}$ and $M A R E_{p, q}$, which are printed in red, correspond to both of the above formulas. From these numerical results, it appears clearly that there exists some symmetry about the first diagonal. This symmetry is defined for $H B R_{p, q}$ by the change of $R$ to $L$ when $p$ is changed in $q$, and vice versa. Moreover, if $M A R E_{p, q}=[\alpha, \beta]$, then $M A R E_{q, p}=[\beta, \alpha]$.

\section{Conclusion}

In this article, the hidden bifurcation routes in multiscroll chaotic attractors generated by saturated function series have been explored. Such hidden bifurcations were first discovered few years ago for Chua multiscroll attractors, which depend on a discrete parameter [2]. Embedding systems of generating multiscrolls in a family of systems possessing a continuous parameter, and using a method similar to the one introduced by Leonov et al. [6,7], the method used for Chua attractors is applicable also to other systems generating multiscroll attractors. In the case of attractors generated by saturated function series, the existence of hidden bifurcation routes have been characterized by the maximal range extension of their attractors and coding of the appearance order of the scrolls, under the control of two parameters. Moreover, it has been confirmed that these routes have interesting symmetries with respect the two parameters. The novelty that this article introduces, is firstly the paradigm of MARE and the formula giving their approximate value depending upon parameters $p$ and $q$, which is linked to the size of the scrolls; secondly the coding of the HBR which is defined for the first time including the basic cell ; and thirdly unearthing the symmetries of these routes, allowing to obtain their coding without any numerical computation.

\section{References}

[1] Lü, J. \& Chen. G. (2006). Generating multiscroll chaotic attractors: Theories, methods and applications. International Journal of Bifurcation and Chaos,16(4), 775-858. 


\begin{tabular}{|c|c|c|c|c|c|c|}
\hline $\mathrm{p}$ & 0 & 1 & 2 & 3 & 4 & 5 \\
\hline 0 & & $\begin{array}{c}{[-40,20] /} \\
\mathrm{B} / \mathrm{L}\end{array}$ & $\begin{array}{c}{[-60,20] /} \\
\mathrm{B} / \mathrm{L} / \mathrm{L}\end{array}$ & $\begin{array}{l}{[-80,20] /} \\
\mathrm{B} / \mathrm{L} / \mathrm{L} / \mathrm{L}\end{array}$ & $\begin{array}{c}{[-100,20] /} \\
B / L / L / L / L\end{array}$ & $\begin{array}{l}{[-120,20] /} \\
B / L / L / L / L / L\end{array}$ \\
\hline 1 & $\begin{array}{c}{[-20,40] /} \\
\mathrm{B} / \mathrm{R}\end{array}$ & $\begin{array}{l}{[-40,40] /} \\
B / 2 \text { Sym }\end{array}$ & $\begin{array}{c}{[-60,40] /} \\
B / 2 S y m / L\end{array}$ & $\begin{array}{c}{[-80,40] /} \\
\mathrm{B} / 2 \mathrm{Sym} / \mathrm{L} / \mathrm{L}\end{array}$ & $\begin{array}{c}{[-100,40] /} \\
\mathrm{B} / 2 \mathrm{Sym} / \mathrm{L} / \mathrm{L} / \mathrm{L}\end{array}$ & $\begin{array}{l}{[-120,40] /} \\
B / 2 S y m / L^{4}\end{array}$ \\
\hline 2 & $\begin{array}{c}{[-20,60] /} \\
\mathrm{B} / \mathrm{R} / \mathrm{R}\end{array}$ & $\begin{array}{c}{[-40,60] /} \\
\mathrm{B} / 2 \mathrm{Sym} / \mathrm{R}\end{array}$ & $\begin{array}{c}{[-60,60] /} \\
\mathrm{B} / 2 \mathrm{Sym} / 2 \mathrm{Sym}\end{array}$ & $\begin{array}{c}{[-80,60] /} \\
\mathrm{B} / 2 \mathrm{Sym} / 2 \mathrm{Sym} / \mathrm{L}\end{array}$ & $\begin{array}{c}{[-100,60] /} \\
B / 2 \operatorname{Sym}^{2} / L^{2}\end{array}$ & $\begin{array}{l}{[-120,60] /} \\
B / 2 s^{2} m^{2} / L^{3}\end{array}$ \\
\hline 3 & $\begin{array}{c}{[-20,80] /} \\
\mathrm{B} / \mathrm{R} / \mathrm{R} / \mathrm{R}\end{array}$ & $\begin{array}{c}{[-40,80] /} \\
\mathrm{B} / 2 \mathrm{Sym} / \mathrm{R} / \mathrm{R}\end{array}$ & $\begin{array}{c}{[-60,80] /} \\
\text { B } 2 \text { Sym/2Sym } / R\end{array}$ & $\begin{array}{l}{[-80,80] /} \\
\mathrm{B} / 2 \text { Sym }^{3}\end{array}$ & $\begin{array}{c}{[-100,80] /} \\
B / 2 \text { Sym }^{3} / L^{1}\end{array}$ & $\begin{array}{l}{[-120,80] /} \\
B / 2 S y m^{3} / L^{2}\end{array}$ \\
\hline 4 & $\begin{array}{c}{[-20,100]} \\
B / R / R / R / R\end{array}$ & $\begin{array}{c}{[-40,100] /} \\
\mathrm{R} / 2 \mathrm{Sym} / \mathrm{R} / \mathrm{R} / \mathrm{R}\end{array}$ & $\begin{array}{c}{[-60,100] /} \\
B / 2 \text { Sym }^{2} / R^{2}\end{array}$ & $\begin{array}{c}{[-80,100]} \\
B / 2 \text { Sym }^{3} / R^{1}\end{array}$ & $\begin{array}{l}{[-100,100]} \\
\mathrm{B} / 2 \operatorname{Sym}^{4}\end{array}$ & $\begin{array}{l}{[-120,100]} \\
\mathrm{B} / 2 S y m^{5} / L^{1}\end{array}$ \\
\hline 5 & $\begin{array}{c}{[-20,120]} \\
B / R / R / R / R / R\end{array}$ & $\begin{array}{c}{[-40,120]} \\
B / 2 \text { Sym }^{1} / R^{4}\end{array}$ & $\begin{array}{c}{[-60,120]} \\
B / 2 \text { Sym }^{2} / R^{3}\end{array}$ & $\begin{array}{l}{[-80,120]} \\
B / 2 S y m^{2} / R^{2}\end{array}$ & $\begin{array}{l}{[-100,120]} \\
\text { B/2Sym }{ }^{4} / R^{1}\end{array}$ & $\begin{array}{l}{[-120,120]} \\
\text { B/2Sym }\end{array}$ \\
\hline
\end{tabular}

Tab. 3: Symmetries of the hidden bifurcation routes : $H B R_{p, q}$ and $M A R E_{p, q}$, numerically computed (black) and inferred from Eqs. (15) and (16) (red).

[2] Menacer, T., Lozi, R. \& Chua, L.O. (2016). Hidden bifurcations in the multispiral Chua attractor. International Journal of Bifurcation and Chaos,16(4), 1630039-1630065.

[3] Dudkowski, D., Jafari, S., Kapitaniak, T., Kuznetsov, N. V., Leonov, G. A. \& Prasad, A. (2016). Hidden attractors in dynamical systems. Physics Reports, 637, 1-50.

[4] Deng, Q. \& Wang, C. (2019). Multi-scroll hidden attractors with two stable equilibrium points. Chaos 29, 093112.

[5] Zhang, X., \& Wang C. (2019), Multiscroll Hyperchaotic System with Hidden Attractors and Its Circuit Implementation. International Journal of Bifurcation and Chaos, 29 (09), 1950117.

[6] Leonov, G. A., \& Kuznetsov, N. V. (2011). Localization of hidden Chua's attractors. Phys. Lett. A, 375, 2230-2233.

[7] Leonov, G. A. (2010). Effective methods for periodic oscillations search in dynamical systems. Appl. Math. Mech, 74, 37-73.

[8] Lü, J., Chen, G., Yu, X., \& Leung, H. (2004). Design and analysis of multiscroll chaotic attractors from saturated function series. IEEE Trans. Circuits Syst. I, 51(12), 2476-2490.
[9] Suykens, J. A., \& Vandewalle, J. (1993). Generation of $\mathrm{n}$-double scrolls $(\mathrm{n}=1 ; 2 ; 3$; $4 ;$. . .). IEEE Trans. Circuits Syst. 40(I), 861-867.

[10] Suykens, J. A., \& Oberoi, R. (1997). A family of n-scroll attractors from a generalized Chua's circuit. Int. J. Electron. Commun, 51(3), 131-138.

[11] Yalcin, M. E., Ozogus, J. A. S., Suykens, J. A. K., \& Vadewalle, J. (2001). n-scroll chaos generators: A simple circuit model. Electron. Lett, 37(3), 147-148.

[12] Leonov, G. A. Vagaitaev, V. I., \& Kuznetsov, N. V. (2010). Algorithm for localizing Chua attractors based on the harmonic linearization method. Dokl. Math, D, 663-666.

[13] Leonov, G. A., \& Kuznetsov, N. V. (2011). Analytical numerical methods for investigation of hidden oscillations in nonlinear control systems. Proc. 18th IFAC World Congress, Milano, Italy, August,28, 2494-2505.

[14] Leonov, G. A., Kuznetsov, N. V. \& Vagaitaev, V. I. (2012). Hidden attractor in smooth Chua systems. Physica D, 241, 1482-1486. 


\section{A Analytical-numerical method for hidden attractor localization}

Recently, the new concepts of self-excited and hidden attractors have been introduced [7], [12], [14]. An attractor is called a self-excited attractor if its basin of attraction overlaps with neighborhood of an equilibrium point; otherwise it is called a hidden attractor. For example, hidden attractors are attractors in systems with no equilibria, or with only one stable equilibrium, or with infinitely many equilibria. A hidden attractor is characterized by its basin of attraction, which does not intersect with small neighborhoods of any equilibrium points, thereby making it very difficult to find.

The name hidden comes from this computational difficulty. However, Leonov et al. [6], [13], [14] found a method to reveal numerically their existence. They develop this method especially for Chua attractor. The method developed in [2], revealing hidden bifurcations in the multispiral Chua attractor, in the case where the parameter of bifurcation which determines the number of spirals is discrete, is based on the method of Leonov et al. [6]

To recall this numerical method, consider a system with one scalar nonlinearity:

$$
\frac{d X}{d t}=M X+\mu \psi\left(\nu^{T} X\right), \quad X \in \mathbb{R}^{3} .
$$

were $M$ is a constant $(n \times n)-$ matrix, $\mu, \nu$ are constant $n$-dimensional vectors, $T$ is a transposition operation, $\psi(\sigma)$ is a continuous piecewisedifferentiable scalar function, and $\psi(0)=0$. Define coefficient $k$ of harmonic linearization in such way that the matrix

$$
M_{0}=M+k \mu \nu^{T}
$$

has a pair of purely imaginary eigenvalues $\pm i \omega_{0}$ $\left(\omega_{0}>0\right)$ and the rest of its eigenvalues have negative real parts. Assume that such $k_{0}$ exists. Then, rewrite system (17) as

$$
\frac{d X}{d t}=M_{0} X+\mu \varphi\left(\nu^{t} X\right)
$$

were $\varphi(\sigma)=\psi(\sigma)-k_{0} \sigma$, and introduce a finite sequence of functions, $\varphi^{0}(\sigma), \varphi^{1}(\sigma), \cdots, \varphi^{m}(\sigma)$, such that the graphs of neighboring function $\varphi^{j}(\sigma)$ and $\varphi^{j+1}(\sigma), j=0, \cdots, m-1$, slightly differ from each other. Suppose that the function $\varphi^{0}(\sigma)$ is small, and $\varphi^{m}(\sigma)=\varphi(\sigma)$. Using the smallness of the function $\varphi^{0}$, one can apply the method of harmonic linearization (describing function method) for the system

$$
\frac{d X}{d t}=M_{0} X+\mu \varphi^{0}\left(\nu^{t} X\right)
$$

and determine a stable nontrivial periodic solution $X^{0}(t)$.

For the localization of the attractor of the original system (19), perform numerically the transformation of this periodic solution. All the points of this stable periodic solution are located in the domain of attraction of the stable periodic solution $X^{1}(t)$ of the system

$$
\frac{d X}{d t}=M_{0} X+\varphi^{j}\left(\nu^{T} X\right)
$$

With $j=1$, or when passing from (20) to system (21) with $j=1$, one can observe the instability bifurcation destroying the periodic solution. In the first case, it is possible to find $X^{1}(t)$ numerically, taking as initial condition of system (21) with $j=1$, any point of the stable periodic solution $X^{0}(t)$.

Starting from this initial condition, after a transient phase, the trajectory reaches the periodic solution $X^{1}(t)$.

Then, after the computation of $X^{1}(t)$, it is possible to obtain a periodic trajectory $X^{2}(t)$ of system (21) with $j=2$, starting from any point of the stable periodic solution $X^{1}(t)$, and so on, to obtain a periodic solution of system (19) if such solution exists.

Remark 2. In some cases it is not possible to get such solution because one observes at a certain step an instability bifurcation destroying the periodic solution.

Remark 3. In the cases of Chua attractor, the periodic solution close to the harmonic one is transformed to a chaotic attractor. This is also the case for multiscroll chaotic attractors from saturated function series, studied in this article. 
In order to determine the initial condition $X^{0}(0)$ of the periodic solution, system (20) can be transformed by a linear nonsingular transformation $S(X=S Y)$ to the form

$$
\left\{\begin{array}{l}
\dot{y_{1}}=-\omega_{0} y_{2}+b_{1} \varphi^{0}\left(y_{1}+c_{3}^{t} Y_{3}\right) \\
\dot{y_{2}}=\omega_{0} y_{1}+b_{2} \varphi^{0}\left(y_{1}+c_{3}^{t} Y_{3}\right) \\
\dot{Y_{3}}=A_{3} Y_{3}+B_{3} \varphi^{0}\left(y_{1}+c_{3}^{t} Y_{3}\right)
\end{array}\right.
$$

Here, $y_{1}, y_{2}$ are scalar values; $Y_{3}$ is an $(n-2)$-dimensional vector, $B_{3}$ and $c_{3}$ are $(n-2)$-dimensional vectors, $b_{1}$ and $b_{2}$ are real numbers; $A_{3}$ is an $(n-2) \times(n-2)$ matrix, where all of its eigenvalues have negative real parts.

Without loss of generality, assume that, for the matrix $A_{3}$, there exists a $d_{2}>0$ such that

$$
Y_{3}^{t}\left(A_{3}+A_{3}^{t}\right) Y_{3} \leq-2 d_{2}\left|Y_{3}\right|^{2} \forall Y_{3} \in \mathbb{R}^{n-2} .
$$

In the scalar case, introduce the describing function $\Phi$ of a real variable $\eta$ as follows:

$$
\Phi(\eta)=\int_{0}^{2 \pi / \omega_{0}} \phi\left(\cos \left(\omega_{0} t\right)(\eta)\right) \cos \left(\omega_{0} t\right) d t
$$

Theorem 1. [12] If a positive $\eta_{0}$ satisfies that

$$
\Phi\left(\eta_{0}\right)=0,\left.b_{1} \frac{d \Phi(\eta)}{d \eta}\right|_{\eta=\eta_{0}}<0
$$

then, for the initial condition of the periodic solution $X^{0}(0)=S\left(y_{1}(0), y_{2}(0), Y_{3}(0)\right)^{T}$ at the first step of algorithm, one has

$$
y_{1}(0)=\eta_{0}+O(\epsilon), y_{2}(0)=0, Y_{3}(0)=O_{n-2}(\epsilon)
$$

were $O_{n-2}(\epsilon)$ is an $(n-2)$-dimensional vector in which all its components are $O(\epsilon)$.

Recall that the stability is defined in the sense that, for all solutions with the initial data sufficiently close to $X^{0}(0)$, the modulus of their difference with $X^{0}(t)$ is uniformly bounded for all $t>0$. For the stability of $X^{0}(t)$, it is sufficient to require the following condition:

$$
\left.b_{1} \frac{d \Phi(\eta)}{d \eta}\right|_{\eta=\eta_{0}}<0 .
$$

In practice, to determine $k_{0}$ and $\omega_{0}$, one uses the transfer function of system (17),

$$
W(\lambda)=r^{T}(M-\lambda I)^{-1} \mu
$$

where $\lambda$ is a complex variable. The number $\omega_{0}$ is determined from the equation $\operatorname{Im} W\left(i \omega_{0}\right)=0$ and $k_{0}$ is then calculated using the formula $k_{0}=$ $-\operatorname{ReW}\left(i \omega_{0}\right)$.

\section{About Authors}

Faiza ZAAMOUNE is currently a thirdyear $\mathrm{PhD}$ student in chaotic systems at LMA Laboratory, University of Biskra, Algeria. She is working under the supervision of $\mathrm{Dr}$ Tidjani Menacer on hidden bifurcations in chaotic systems. She holds a Master Degree from University of Biskra, Algeria. Her major research interest is in hidden attractors and hidden bifurcations.

Tidjani MENACER is a professor class A lecturer at the University of Biskra, Algeria. He completed his PhD thesis in Algeria under the supervision of Prof. Nasr-Edine Hamri in 2014. In 2016, he became a Professor of Ability to derive research. He has published some papers and participated in many local and international conferences. $\mathrm{He}$ is reviewer for many journals. Nowadays, his research areas include dynamical systems, bifurcation, control of chaos, synchronization of chaos, fractional chaotic systems, and recently hidden attractors and hidden bifurcations.

René LOZI is Emeritus Professor at at University Côte d'Azur, Dieudonné Center of Mathematics, France. He completed the PhD degree with his French State Thesis (on chaotic dynamical systems) under the supervision of Prof. René Thom (Fields medallist) in 1983. In 1991, he became Full Professor at University of Nice and IUFM (Institute for teacher trainees). He has served as Director of this institute (2001-2006) and as Vice-Chairman of the French Board of Directors of IUFM (2004-2006). He is member of several editorial boards of international journals. In 1977, he discovered a particular mapping of the plane 
having a strange attractor (now, commonly known as "Lozi map"). Nowadays, his research areas include complexity and emergence theory, dynamical systems, bifurcations, control of chaos, cryptography based on chaos, and recently memristors (physical devices for neurocomputing). He is working in those fields with renowned researchers from many countries. He received the Dr. Zakir Husain Award 2012 from the Indian Society of Industrial and Applied Mathematics during the 12th biannual conference of ISIAM at the University of Punjab, Patialia, January 2015.
Guanrong CHEN is a Chair Professor and the Director of the "Centre for Chaos and Complex Networks" at the City University of Hong Kong. He was elected IEEE Fellow in 1997, awarded the 2011 Euler Gold Medal from Russia, and conferred Honorary Doctor Degrees by the Saint Petersburg State University, Russia in 2011 and by the University of Normandy, France in 2014. He is a Highly Cited Researcher in Engineering (since 2009) according to Thomson Reuters. He is a Member of the Academy of Europe (since 2014) and a Fellow of The World Academy of Sciences (since 2015). 